\title{
Análise da qualidade de vida de portadores de uveítes de causas infecciosas e não infecciosas pelo questionário NEI-VFQ-25
}

\author{
Analysis of the life quality of infectious and non-infectious patients with \\ uveitis using the NEI-VFQ-25 questionnaire
}

\author{
Paula Resende Aquino de Assis Pereira Mello ${ }^{1}$ \\ Adriano de Carvalho Roma ${ }^{2}$ \\ Haroldo Vieira Moraes Júnior ${ }^{3}$
}

Trabalho realizado no Departamento de Oftalmologia do Hospital Universitário Clementino Fraga Filho Universidade Federal do Rio de Janeiro - UFRJ - Rio de Janeiro (RJ) - Brasil.

${ }^{1}$ Pós-graduanda nível mestrado do Serviço de Oftalmologia do Hospital Universitário Clementino Fraga Filho da Universidade Federal do Rio de Janeiro - UFRJ - Rio de Janeiro (RJ) - Brasil.

${ }^{2}$ Mestre, Médico colaborador do Serviço de Oftalmologia do Hospital Universitário Clementino Fraga Filho da UFRJ - Rio de Janeiro (RJ) - Brasil.

Professor adjunto e chefe do Setor de Uveíte do Serviço de Oftalmologia do Hospital Universitário Clementino Fraga Filho da UFRJ - Rio de Janeiro (RJ) - Brasil

Endereço para correspondênica: Paula Resende Aquino de Assis Pereira Mello. Rua Desembargador Canedo, 375 - Apto. 502 - Muriaé (MG)

CEP 36880-000

E-mail: paula.raap@gmail.com

Recebido para publicação em 30.09.2007

Última versão recebida em 16.06.2008

Aprovação em 14.08.2008

Nota Editorial: Depois de concluída a análise do artigo sob sigilo editorial e com a anuência dos Drs. Carlos Roberto Neufeld e Moysés Eduardo Zajdenweber sobre a divulgação de seus nomes como revisores, agradecemos suas participações neste processo.

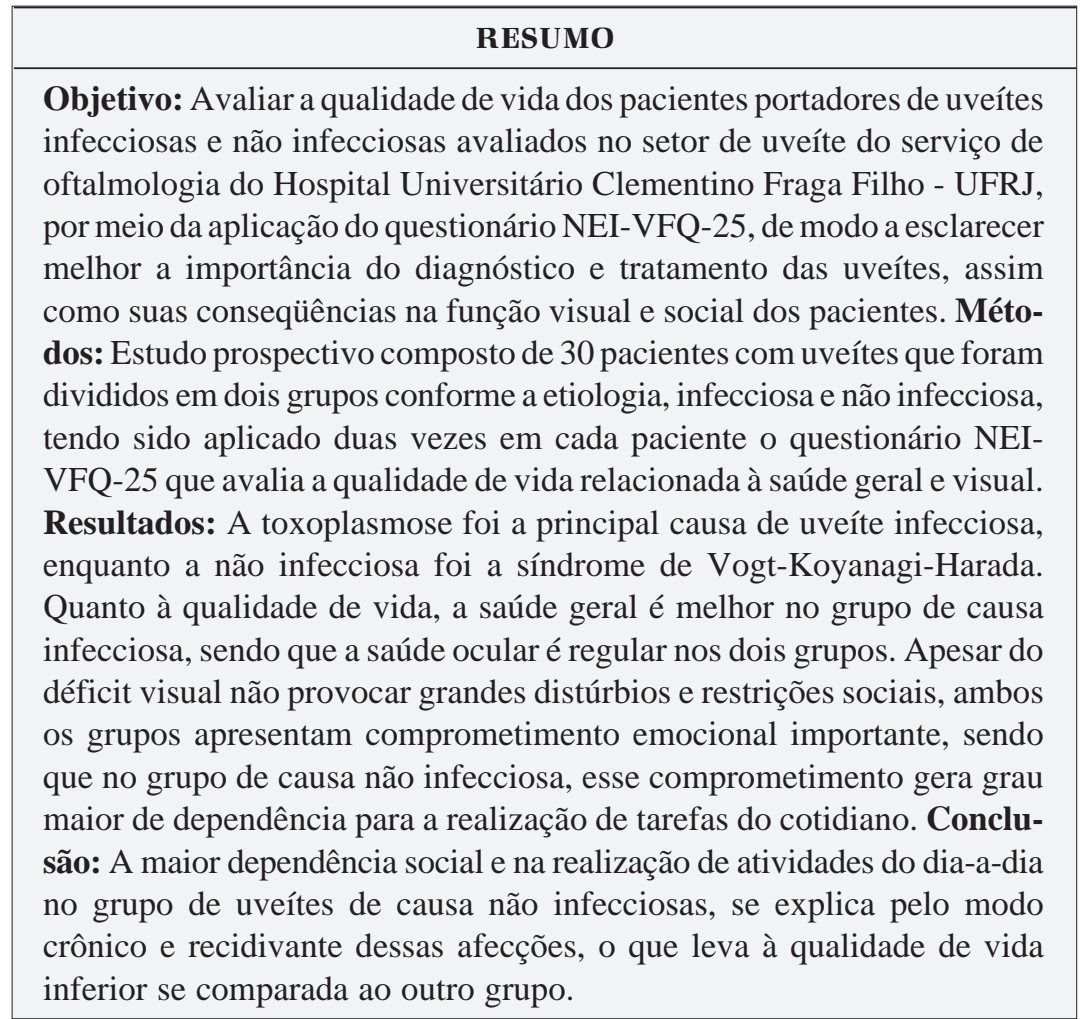

Descritores: Qualidade de vida; Saúde ocular; Questionários; Uveítes/etiologia; Infecções oculares

\section{INTRODUCÃ̃O}

Considerando a importância da capacidade visual do indivíduo para a sua independência na realização de suas atividades cotidianas e sobrevivência no mundo, torna-se importante a avaliação da qualidade de vida de pessoas portadoras de alguma patologia que provoque comprometimento visual $^{(1-3)}$.

Visto que a maioria dos médicos se preocupa mais com o fator doença e os meios para que se alcance a cura, não é dada importância às consequiências socioeconômicas e psicológicas que o déficit visual determina na vida do doente, assim como muitas vezes, este também se preocupa apenas em ficar curado, não se importando com as suas futuras restrições ${ }^{(2-6)}$. 
“Alguns pacientes estão mais preocupados com os episódios recorrentes da inflamação e a necessidade do uso de medicações freqüentes. Outros estão concentrados no resultado visual final, mesmo se a inflamação for leve, não se importando com a freqüência dos medicamentos"(7-8).

Para tanto, a avaliação da qualidade de vida desses pacientes, permite a otimização de estratégias de tratamento e meios de inseri-los adequadamente à estrutura social a que pertencem $^{(1,4,9)}$. "Somente através do entendimento da relação do paciente com a sua doença, o médico poderá escolher melhor a terapêutica e orientá-lo melhor quanto ao prognóstico, tempo de evolução e possibilidades de recuperação"(7,10-11).

Dentre as diversas patologias que acometem a visão, priorizamos neste estudo as uveítes, que são inflamações que acometem o trato uveal (íris, corpo ciliar e coróide) ${ }^{(5,7)}$.

As uveítes podem ser classificadas de diversas maneiras, segundo as estruturas acometidas, o curso clínico (agudo, subagudo ou crônico), a uni ou bilateralidade, a etiologia e o aspecto clínico como se apresentam ${ }^{(7)}$.

Os autores dividiram a amostra em dois grupos segundo a causa: infecciosas e não infecciosas.

O questionário de função visual (VFQ) foi desenvolvido pelo "National Eye Institute" (NEI) com o objetivo de criar um instrumento para avaliar a influência da incapacidade visual e dos sintomas visuais em domínios de saúde genéricos, tais como, o bem-estar emocional e função visual; em adição a domínios orientados para tarefas relacionadas a atividades diárias. Esta avaliação é realizada por meio de perguntas que determinam o grau de dificuldade para executar determinadas tarefas, como: descer escadas, ler placas na rua, assim como outras atividades do cotidiano ${ }^{(2,3,9-11,14-18)}$.

O questionário de função visual NEI-VFQ-25 é um produto de análise e redução de itens do NEI-VFQ-51, realizada com a finalidade de diminuir o tempo de administração do questionário, torná-lo menos cansativo para o paciente e mais prático para o uso na rotina diária dos serviços de saúde ${ }^{(14-15)}$.

O objetivo deste estudo foi avaliar a qualidade de vida dos portadores de uveítes infecciosas e não infecciosas por meio da aplicação do questionário NEI-VFQ-25, de modo a esclarecer melhor sobre a importância do diagnóstico e tratamento das uveítes, assim como suas consequiências na função visual e social dos pacientes.

\section{MÉTODOS}

Foram avaliados 30 pacientes atendidos no setor de uveíte do departamento de oftalmologia do Hospital Universitário Clementino Fraga Filho - UFRJ. Todos os pacientes apresentavam o diagnóstico confirmado no período do estudo, por meio de exame oftalmológico e complementar.

Esta pesquisa foi realizada no período de novembro de 2006 a abril de 2007.

O projeto de pesquisa foi aprovado pelo Comitê de Ética em Pesquisa em Seres Humanos do Hospital acima referido e devidamente registrado na Comissão Nacional de Ética em Pesquisa.

Os 30 pacientes incluídos no estudo foram divididos em dois grupos: portadores de uveítes infecciosas e uveítes não infecciosas. Treze pacientes foram do grupo de causas infecciosas e 17 do grupo de causas não infecciosas. Em relação ao grupo de uveítes infecciosas, nove casos apresentaram o diagnóstico de toxoplasmose e o restante da amostra com um caso de cada diagnóstico: necrose retiniana aguda em pacientes HIV positivos, retinite por citomegalovírus, tuberculose ocular e coroidite multifocal. Quanto ao grupo de uveítes não infecciosas, seis casos apresentaram diagnóstico de VogtKoyanagi-Harada, cinco de artrite reumatóide juvenil, quatro com doença de Behçet, um caso do esclerite e um com retocolite ulcerativa.

Foi aplicado o questionário National Eye Institute - Visual Function Questionnaire - 25 (NEI-VFQ-25), que avalia a saúde geral do paciente, assim como a qualidade de vida relacionada à saúde visual (Anexo 1).

Este questionário tem validade e confiabilidade estatisticamente comprovados ${ }^{(14)}$.

O questionário é composto de três partes, sendo a primeira com quatro perguntas relacionada com a saúde geral e visão. A segunda parte é formada por doze perguntas sobre dificuldades em realizar algumas atividades e a terceira parte com nove perguntas sobre como as coisas que são feitas podem ser afetadas pela visão. O mesmo foi aplicado duas vezes em cada paciente com intervalo mínimo de 15 dias, sendo que, em caso de grande discrepância entre as respostas da primeira e da segunda aplicação, o mesmo foi considerado inválido. As respostas são numeradas de um até seis, sendo considerada inválida aquelas que tiveram variação maior ou igual a 3 . Havendo pequena diferença (variação menor ou igual a 2), considera-se a última resposta para fins estatísticos.

Foram colhidos os seguintes dados: sexo, idade, escolaridade, profissão, procedência. Todos os pacientes foram orientados quanto aos objetivos e a metodologia empregada, assinando após esta orientação, o termo de consentimento livre e esclarecido.

Após a consulta ao serviço de Bioestatística, para a obtenção dos resultados foi decidido pela análise estatística descritiva.

\section{RESULTADOS}

Dos 30 pacientes avaliados, 13 foram do grupo de uveítes infecciosas $(43,33 \%)$ e 17 do grupo de uveítes não infecciosas $(56,66 \%)$.

Entre os pacientes com diagnóstico de uveíte infecciosa, 6 $(46,15 \%)$ foram do sexo masculino e $7(53,84 \%)$ do sexo feminino. A média de idade foi de 34,15 anos, variando de 16 a 62 anos. Nove $(69,23 \%)$ pacientes foram brancos, $3(23,07 \%)$ pardos e $1(7,69 \%)$ negro.

Quanto à procedência, 7 (53,84\%) foram do Rio de Janeiro e $6(46,15 \%)$ de outros estados. 
Em relação à escolaridade, $3(23,07 \%)$ tinham segundo grau completo, $3(23,07 \%)$ segundo grau incompleto, $5(38,46 \%)$ primeiro grau incompleto, $1(7,69 \%)$ primeiro grau completo e $1(7,69 \%)$ analfabeto.

Em relação ao grupo com uveítes não infecciosas, 6 $(35,29 \%)$ foram do sexo masculino, $11(64,70 \%)$ do sexo feminino. A média de idade foi de 33,05 anos variando de 7 a 70 anos.

Dez $(58,82 \%)$ pacientes foram brancos, $6(35,29 \%)$ pardos e $1(5,88 \%)$ negro.

Quanto à procedência, $16(94,11 \%)$ foram do Rio de Janeiro e $1(5,88 \%)$ de outro estado.

Em relação à escolaridade, $5(29,41 \%)$ tinham segundo grau completo, $2(11,76 \%)$ segundo grau incompleto, 2 $(11,76 \%)$ primeiro grau completo, $8(47,05 \%)$ primeiro grau incompleto e nenhum analfabeto.

No quadro 1 temos os resultados do questionário VFQ-25.

\section{DISCUSSÃO}

No grupo estudado, constatamos que a principal causa de uveítes infecciosas foi a toxoplasmose, e entre as não infecciosas foi a síndrome de Vogt-Koyanagi-Harada.

Nossos resultados estão de acordo com os achados da literatura. A causa mais comum das uveítes infecciosas posteriores é a toxoplasmose, acometendo $80 \%$ dos casos no Brasil, e a síndrome de Vogt-Koyanagi-Harada é responsável por $2 \%$ a $4 \%$ das uveítes, sendo a principal causa de uveítes não infecciosas ${ }^{(7,19-20)}$.

Observamos que a prevalência, tanto nas uveítes infecciosas quanto nas não infecciosas foi maior no sexo feminino, sendo a maior parte branca, com primeiro grau incompleto e média de idade de trinta e três anos $(33,6)$. Estes resultados quanto ao sexo e idade média relacionados às uveítes não

\begin{tabular}{|c|c|c|c|c|c|}
\hline \multicolumn{6}{|c|}{ Quadro 1. Resultados obtidos } \\
\hline Variável & Respostas & $\begin{array}{c}\text { Número de } \\
\text { pacientes uveítes } \\
\text { não infecciosas }\end{array}$ & $\begin{array}{l}\text { Respostas } \\
\text { inválidas }\end{array}$ & $\begin{array}{l}\text { Número de } \\
\text { pacientes uveítes } \\
\text { infecciosas }\end{array}$ & $\begin{array}{l}\text { Respostas } \\
\text { inválidas }\end{array}$ \\
\hline VFQ-25 & Muito boa & $1(5,88 \%)$ & \multirow{3}{*}{0} & $3(23,07 \%)$ & \multirow{3}{*}{0} \\
\hline \multirow[t]{2}{*}{ Saúde geral } & Boa & 7 (41,17\%) & & $6(46,15 \%)$ & \\
\hline & Não satisfatória & 9 (52,94\%) & & $4(30,76 \%)$ & \\
\hline VFQ-25 & Boa & $1(5,88 \%)$ & \multirow{3}{*}{0} & $4(30,76 \%)$ & \multirow{3}{*}{0} \\
\hline \multirow[t]{2}{*}{ Saúde ocular } & Regular & $11(64,70 \%)$ & & 5 (38,46\%) & \\
\hline & Ruim & $5(29,42 \%)$ & & $4(30,76 \%)$ & \\
\hline VFQ-25 & Sim & $12(70,58 \%)$ & \multirow{2}{*}{0} & $8(61,53 \%)$ & \multirow{2}{*}{0} \\
\hline Dor ocular & Não & $5(29,42 \%)$ & & 5 (38,46\%) & \\
\hline VFQ-25 & Pouca & $3(17,64 \%)$ & \multirow{4}{*}{0} & $2(15,38 \%)$ & \multirow{4}{*}{3} \\
\hline Dificuldade & Moderada & 4 (23,52\%) & & 2 (15,38\%) & \\
\hline \multirow[t]{2}{*}{ para longe } & Muita & $6(35,29 \%)$ & & $3(23,07 \%)$ & \\
\hline & Não & $4(23,52 \%)$ & & $3(23,07 \%)$ & \\
\hline VFQ-25 & Pouca & $3(17,64 \%)$ & \multirow{4}{*}{1} & $3(23,07 \%)$ & \multirow{4}{*}{2} \\
\hline Dificuldade & Moderada & $4(23,52 \%)$ & & $1(7,69 \%)$ & \\
\hline \multirow[t]{2}{*}{ para leitura } & Muita & $5(29,42 \%)$ & & $5(38,46 \%)$ & \\
\hline & Não & $4(23,52 \%)$ & & $2(15,38 \%)$ & \\
\hline VFQ-25 & Pouca & $3(17,64 \%)$ & \multirow{4}{*}{0} & $3(23,07 \%)$ & \multirow{4}{*}{2} \\
\hline \multirow[t]{3}{*}{ Atividade perto } & Moderada & $3(17,64 \%)$ & & $2(15,38 \%)$ & \\
\hline & Muita & $5(29,42 \%)$ & & $3(23,07 \%)$ & \\
\hline & Não & $6(35,29 \%)$ & & $3(23,07 \%)$ & \\
\hline VFQ-25 & Sim & $8(47,05 \%)$ & \multirow{2}{*}{0} & $2(15,38 \%)$ & \multirow{2}{*}{1} \\
\hline Visão de cores & Não & 9 (52,94\%) & & 10 (76,92\%) & \\
\hline VFQ-25 & Sim & $11(64,70 \%)$ & \multirow{2}{*}{0} & 7 (53,84\%) & \multirow{2}{*}{0} \\
\hline Visão periférica & Não & 6 (35,29\%) & & $6(46,15 \%)$ & \\
\hline VFQ-25 & Pouca & $1(5,88 \%)$ & \multirow{4}{*}{2} & 0 & \multirow{4}{*}{2} \\
\hline \multirow[t]{3}{*}{ Social } & Moderada & 0 & & 0 & \\
\hline & Muita & 0 & & $1(7,69 \%)$ & \\
\hline & Não & $14(82,35 \%)$ & & $10(76,38 \%)$ & \\
\hline VFQ-25 & Sim & $13(76,47 \%)$ & \multirow[b]{2}{*}{0} & 7 (53,84\%) & \multirow[b]{2}{*}{0} \\
\hline Atividades diárias & Não & $4(23,52 \%)$ & & $6(46,15 \%)$ & \\
\hline VFQ-25 & Sim & $9(52,94 \%)$ & \multirow[b]{2}{*}{1} & $3(23,07 \%)$ & \\
\hline Dependência & Não & 7 (41,17\%) & & $10(76,92 \%)$ & 0 \\
\hline VFQ-25 & Sim & $15(88,23 \%)$ & & $8(61,53 \%)$ & \\
\hline Mental & Não & $2(11,76 \%)$ & 0 & $4(30,76 \%)$ & 1 \\
\hline
\end{tabular}


infecciosas, equivalem-se aos achados da literatura ${ }^{(14,21)}$. Em relação à principal causa de uveítes infecciosas, a faixa etária acometida varia entre a $1^{\underline{a}}$ e $2^{\underline{a}}$ décadas de $\operatorname{vida}^{(7,21)}$.

No nosso trabalho, a prevalência foi maior na quarta década de vida. Esta diferença foi devido a não investigação da origem, congênita ou adquirida, da doença. Pois, os pacientes do estudo já estavam com o diagnóstico estabelecido, o que impediu a diferenciação entre a causa congênita ou adquirida.

Comparando a qualidade de vida entre os grupos, percebemos que os pacientes com uveítes de causas infecciosas consideram a saúde geral boa, e os de causas não infecciosas, insatisfatória. Como a saúde geral está diretamente relacionada à saúde ocular, o curso da doença assim como o tempo de duração da atividade inflamatória e o número de recorrência são condições determinantes para o surgimento de complicações, o que justifica os nossos resultados ${ }^{(22-24)}$.

Quanto à saúde ocular, o resultado foi regular para ambos os grupos, com presença de dor ocular na maioria dos casos.

Grande parte dos pacientes, nos dois grupos, relatou muita dificuldade com as atividades que requerem boa visão para longe e perto. Esta dificuldade pode estar relacionada não apenas à uveíte, mas também a outras doenças associadas, como erro refracional e catarata.

Nos dois grupos analisados, a maioria não apresentou alterações na distinção de cores, enquanto que relacionado à visão periférica, grande parte apresentou alterações, sendo sutis no grupo de uveítes infecciosas e de moderadas a significativa no outro grupo.

Quanto aos aspectos sociais e psicológicos, observamos que o acometimento visual, dependendo de sua etiologia, pode provocar restrições em suas vidas. No entanto, observamos que nos casos de etiologias não infecciosas, a maioria apresenta dependência nas suas atividades diárias.

Analisando o questionário, notamos um maior número de respostas inválidas no grupo de uveítes infecciosas, portanto, não foi possível justificar este resultado pelas características da doença, já que os pacientes do estudo eram submetidos ao acompanhamento clínico de rotina e nenhum deles apresentou doença em atividade durante o período que foi realizado a pesquisa, não havendo mudança da acuidade visual que pudesse interferir na qualidade de vida, ou por outros motivos, como o nível intelectual dos pacientes.

Em relação ao grupo das uveítes não infecciosas, foram poucas as respostas inválidas, não havendo também, alteração do curso clínico da doença no período estudado, não interferindo na qualidade de vida. Portanto, não foi possível encontrar uma explicação que justificasse a diferença de respostas inválidas entre os grupos.

Desse modo, vemos que as dependências sociais e a realização das atividades diárias é menos observada entre os portadores de uveítes infecciosas se comparado aos portadores de uveítes não infecciosas. Fato este que se explica pelo comportamento recidivante e crônico dessas afecções, ocasionando maior chance de complicações, o que mostra que a qualidade de vida desses pacientes é ligeiramente inferior se comparado ao outro grupo, o que nos faz refletir a respeito de diferentes abordagens e estratégias de tratamento e acompanhamento entre os grupos, assim como, a utilização de futuros cuidados de saúde.

Como as decisões clínicas e cirúrgicas são feitas baseadas apenas nos valores da acuidade visual, e que o mundo real é composto de objetos de diferentes frequiências luminosas e contrastes, a avaliação apenas da acuidade visual é uma forma simples de verificação da qualidade de vida, tornando-se importante a verificação da percepção do paciente de seu próprio desempenho visual.

O questionário NEI-VFQ 25 pode ser usado para avaliar a influência das várias doenças oculares e as intervenções necessárias para o bem-estar do dia-a-dia dos pacientes.

\section{CONCLUSÃO}

Pelo fato crônico e recidivante das uveítes de causas não infecciosas, a qualidade de vida dos pacientes deste grupo torna-se inferior, sendo causada por uma maior dependência social e dificuldade na realização das atividades do dia-a-dia. Portanto, as estratégias de tratamento, deste grupo de pacientes, devem abranger tanto os aspectos de saúde visual quanto a saúde mental.

\section{ABSTRACT}

Purpose: To evaluate the life quality of patients with infectious and non-infectious uveitis evaluated at the uveitis service of the Hospital Universitário Clementino Fraga FilhoUFRJ, using the NEI-VFQ-25 questionnaire in order to clarify the importance of uveitis diagnosis and treatment as well as its consequences to visual and social functions of the patients. Methods: Prospective study of 30 patients with uveitis, who were divided into two groups according to the etiology, infectious and non-infectious, with the NEI-VFQ-25 form that evaluates the life quality regarding general and visual health which was applied twice to each patient. Results: The main cause of infectious uveitis was toxoplasmosis, and of the non-infectious was Vogt-Koyanagi-Harada syndrome. Concerning quality of life, general health is better in the infectious uveitis group, but ocular health is regular in both groups. Although visual deficit does not cause great disturbances and social restrictions, both groups show important emotional alteration, but in the non-infectious group this alteration causes a higher dependence level regarding daily activities. Conclusion: The greater social dependence as well as that regarding performance of daily activities in the uveitis group of non-infectious cause is explained by the chronic and relapsing mode of these affections, which turns life quality lower if compared to the other group.

Keywords: Life quality; Ocular health; Questionnaires; Uveitis/etiology; Eye infections 


\section{REFERÊNCIAS}

1. Gill TM, Feinstein AR. A critical appraisal of the quality of quality-of-life measurements. JAMA. 1994; 272(8):619-26. Comment in: JAMA. 1994; 272(8):630- JAMA. 1995;273(11):843-4; author reply 844-5. JAMA. 1995; 273(11):843; author reply 844-5. JAMA. 1995;273(11):844; author reply 844-5. JAMA. 1995;273(11):844; author reply 844-5.

2. Testa MA, Simonson DC. Assesment of quality-of-life outcomes. N Engl J Med. 1996;334(13):835-40. Comment in: N Engl J Med. 1996;335(7):520-1; author reply 521-2. N Engl J Med. 1996;335(7):521; author reply 521-2. N Engl J Med. 1996;335(7):521; author reply 521-2.

3. Vitale S, Schein OD. Qualitative research in functional vision. Int Ophthalmol Cin. 2003;43(2):17-30.

4. Brown MM, Brown GC, Sharma S, Hollands H, Landy J. Quality of life and systemic comorbidities in patients with ophthalmic disease. $\mathrm{Br} \mathrm{J}$ Ophthalmol. 2002;86(1):8-11.

5. Gardiner AM, Armstrong RA, Dunne MC, Murray PI. Correlation between visual function and visual ability in patients with uveitis. $\mathrm{Br} \mathrm{J}$ Ophthalmol. 2002;86(9):993-6.

6. Globe DR, Warma R, Torres M, Wu J, Klein R, Azen SP; Los Angeles Latino Eye Study Group. Self-reported comorbidities and visual function in a population-based study: the Los Angeles Latino Eye Study. Arch Ophthalmol. 2005;123(6):815-21.

7. Oréfice F. Uveíte clínica e cirúrgica: texto e atlas. $2^{\circ}$ ed. Rio de Janeiro: Cultura Médica; 2005. v.1, p.15-21;45-59.

8. Schiffman RM, Jacobsen G, Whitcup SM. Visual functioning and general health status in patients with uveitis. Arch Ophthalmol. 2001;119(6):841-9.

9. Guyatt GH, Naylor CD, Juniper E, Heyland DK, Jaeschke R, Cook DJ. Users' guides to medical literature. XII. How to use articles about health-related quality of life. Evidence-Based Medicine Working Group. JAMA. 1997; 277(15):1232-7.

10. Stelmack JA, Stelmack TR, Massof RW. Measuring low-vision rehabilitation outcomes with the NEI VFQ-25. Invest Ophthalmol Vis Sci. 2002;43(9): 2859-68.

11. Wolffsohn JS, Cochrane AL, Watt NA. Implementation methods for vision related quality of life questionnaires. Br J Ophthalmol. 2000;84(9):1035-40.

12. Ferraz EVAP, Lima CA, Cella W, Arieta CEL. Adaptação de questionário de avaliação da qualidade de vida para aplicação em portadores de catarata. Arq Bras Ophthalmol. 2002;65(3):293-8.

13. Globe D, Varma R, Azen SP, Paz S, Yu E, Preston-Martin S; Los Angeles Latino Eye Study Group. Psychometric performance of the NEI VFQ-25 in visually normal Latinos: the Los Angeles Latino Eye Study. Invest Ophthalmol Vis Sci. 2003;44(4):1470-8.

14. Madureira RF. Avaliação da qualidade de vida pelo questionário NEI-VFQ-25 nos pacientes portadores da síndrome de Vogt-Koyanagi-Harada [dissertação]. Rio de Janeiro: Universidade Federal do Rio de Janeiro. Faculdade de Medicina; 2005.

15. Mangione CM, Lee PP, Gutierrez PR, Spritzer K, Berry S, Hays RD; Lee PP, Gutierrez PR, Spritzer K, Berry S, Hays RD; National Eye Institute Visual Function Questionnaire Field Test Investigators. Development of the 25-item National Eye Institute Visual Function Questionnaire. Arch Ophthalmol. 2001;119(7):1050-8.

16. Owsley C, McGwin G Jr. Depression and the 25-item National Eye Institute Visual Function Questionnaire in older adults. Ophthalmology. 2004;111(12): 2259-64.

17. Vu HT, Keeffe JE, McCarty CA, Taylor HR. Impact of unilateral and bilateral vision loss on quality of life. Br J Ophthalmol. 2005;89(3):360-3.

18. Bradley EA, Bradley D, Bartley GB. Evaluating health-related quality of life in ophthalmic disease: practical considerations. Arch Ophthalmol. 2006;124(1): 121-2.

19. Abreu MT, Hirata PS, Belfort Júnior R, Domingues Neto S. Uveítes em São Paulo-estudo epidemiológico, clínico e terapêutico. Arq Bras Oftalmol. 1980;43(1):10-6.

20. Tenório G, Sreres MP, Castro FM. Distribución geográfica de las uveítis. In: Belfort Júnior R, Couto CA, Castro FM. Uveítis: sinopsis diagnostica y terapeutica. México: Ciba Vision; 1997. p.31-4.

21. Oréfice F. Uveíte clínica e cirúrgica: texto e atlas. $2^{\circ}$ ed. Rio de Janeiro: Cultura Médica; 2005. v.2, p.709-12;1111-30.

22. Moorthy RS, Inomata H, Rao NA. Vogt-Koyanagi-Harada syndrome. Surv Ophthalmol. 1995;39(4):265-92.

23. Ohno S, Minakawa R, Matsuda H. Clinical studies of Vogt-Koyanagi-Harada's disease. Jpn J Ophthalmol. 1988;32(3):334-43.

24. Read RW, Rechodouni A, Butani N, Johnston R, LaBree LD, Smith RE, Rao NA. Complications and prognostic factors in Vogt-Koyanagi-Harada disease. Am J Ophthalmol. 2001;131(5):599-606.

Anexo 1. Questionário NEI-VFQ-25

PARTE 1 - SAÚDE GERAL E VISÃO

1 - Como você acha que está a sua saúde?

\begin{tabular}{|c|c|}
\hline Excelente ..................... (1) & Muito \\
\hline Boa ............................. (3) & Regule \\
\hline
\end{tabular}

Ruim

* Pule a pergunta 1 quando o VFQ-25 estiver sendo aplicado com o SF-36 ou RAND 36

- Item Pesquisa de Saúde 1.0

2 - Como você acha que está a sua visão (com óculos ou lentes de contato, se usuário)?
Excelente
Regular
Boa
Muito ruim
Ruim
Completamente cego

3 - Você tem se preocupado com sua visão?

Não ............................ (1)
Algumas vezes ............ (3)
O tempo todo .............. (5)

Um pouco

A maior parte do tempo

4 - Você tem sentido dor ou desconforto nos seus olhos (p.ex.: coceira, queimação, dor)? Sim ou não? Esta dor ou desconforto é:

Não sinto .

Fraca

Moderada

Severa 


\section{PARTE 2 - DIFICULDADES COM ATIVIDADES}

As próximas perguntas são sobre dificuldades em fazer algumas atividades, usando seus óculos ou lentes de contato, caso você os use, para as seguintes atividades:

5 - Você tem dificuldade para ler jornal, livro ou revista?

Não tenho dificuldade......

(1) Pouca dificuldade

Dificuldade moderada

(3) Muita dificuldade

Deixou de ler por causa da visão

. (5) Deixou de ler por outros motivos, ou não se interessa por leitura ..

6 - Você tem dificuldade para cozinhar, costurar ou ver coisas de perto?

Não tenho dificuldade

(1) Pouca dificuldade

Dificuldade moderada

(3) Muita dificuldade

Deixou de fazer devido à visão

(5) Deixou de fazer por outros motivos, ou não se interessa por isso .

7 - Por causa da sua visão, você tem tido dificuldade para achar coisas quando se encontram misturadas a outros objetos (talher, sapato, roupa)?

Não tenho dificuldade

(1) Pouca dificuldade

Dificuldade moderada

.. (3) Muita dificuldade

Deixou de fazer devido à visão

(5) Deixou de fazer por outros motivos, ou não se interessa por isso

8 - Você tem dificuldade para ler placas na rua ou letreiro do ônibus?

Não tenho dificuldade...

(1) Pouca dificuldade

Dificuldade moderada

. (3) Muita dificuldade

Deixou de fazer devido à visão

(5) Deixou de fazer por outros motivos, ou não se interessa por isso

9 - Você tem tido dificuldade para descer escadas?

Não tenho dificuldade...

(1) Pouca dificuldade

Dificuldade moderada

. (3) Muita dificuldade

Deixou de fazer devido à visão

(5) Deixou de fazer por outros motivos, ou não se interessa por isso

10 - Você tem tido dificuldade para enxergar os objetos a seu lado quando você está andando sozinho?

Não tenho dificuldade.......

. (1) Pouca dificuldade

Dificuldade moderada

(3) Muita dificuldade

Deixou de fazer devido à visão

(5) Deixou de fazer por outros motivos, ou não se interessa por isso(6)

11 - Você tem dificuldade para conversar com os amigos ou parentes por causa da sua visão?

Não tenho dificuldade

.(1) Pouca dificuldade

Dificuldade moderada

(3) Muita dificuldade

Deixou de fazer devido à visão

(5) Deixou de fazer por outros motivos, ou não se interessa por isso

12 - Você tem dificuldade, por causa da visão, para diferenciar as cores?

Não tenho dificuldade

(1) Pouca dificuldade

Dificuldade moderada

(3) Muita dificuldade

Deixou de trocar de roupa sozinho por causa da visão.(5)

Deixou de fazer por outros motivos, ou não se interessa por isso . (6) 13 - Você tem dificuldade, por causa da visão, para reunir-se com os amigos ou parentes em suas casas, em festas ou em reuniões?

Não tenho dificuldade

. (1) Pouca dificuldade

Dificuldade moderada

(3) Muita dificuldade

Deixou de fazer devido à visão

(5) Deixou de fazer por outros motivos, ou não se interessa por isso

14 - Você tem dificuldade, por causa da visão, para enxergar as pessoas quando estão do outro lado da rua?

Não tenho dificuldade

(1) Pouca dificuldade

Dificuldade moderada

(3) Muita dificuldade

Deixou de assistir por causa da visão

(5) Deixou de assistir por outros motivos, ou não se interessa por isso

15 - Você dirige, mesmo que de vez em quando?

Sim (vá para questão 15c)

Não 
Continuação da Parte 2

15a- Você nunca dirigiu ou desistiu de dirigir?

Nunca dirigiu (vá para parte 3, questão 17)

(1) Desistiu

15b- Se você desistiu, foi devido à visão, por outras razões ou as duas coisas ao mesmo tempo?

Principalmente pela visão (vá para parte 3, questão 17) . (1) Por outros motivos (vá para parte 3, questão 17)....

Pela visão e outros motivos (vá para parte 3, questão 17) .. (3)

15c- Você tem dificuldade para dirigir, durante o dia, em lugares conhecidos?

Não tenho dificuldade.

(1) Pouca dificuldade

Moderara dificuldade

(3) Muita dificuldade

16 - Você tem dificuldade para dirigir durante a noite?

Não tenho dificuldade.

(1) Pouca dificuldade

Dificuldade moderada

(3) Muita dificuldade

Deixou de dirigir devido à visão

(5) Deixou de dirigir por outros motivos, ou não se interessa por isso

\section{PARTE 3 - REAÇÕES AOS PROBLEMAS DE VISÃO}

As próximas perguntas são sobre como as coisas que você faz podem ser afetadas pela sua visão.

17 - Você tem deixado de realizar coisas que gosta por causa da sua visão?
Sempre
A maioria das vezes
De vez em quando..... (3)
Poucas vezes
Nunca

18 - Você se acha limitado para trabalhar ou realizar outras atividades por causa da visão?
Sempre
A maioria das vezes
De vez em quando..... (3)
Poucas vezes
Nunca

19 - Você sente desconforto nos olhos ou em volta deles (por ex.: queimação, coceira, dor) que faz você deixar de fazer coisas que gosta?
Sempre
De vez em quando..... (3)
Nunca

A maioria das vezes

Poucas vezes

20 - Você fica muito tempo em casa por causa da sua visão?
Sempre
De vez em quando..... (3)
Nunca

A maioria das vezes

Poucas vezes

21 - Você tem se sentido triste por causa da sua visão?
Sempre
De vez em quando..... (3)
A maioria das vezes
Poucas vezes

Nunca

22 - Você tem sentido receio de fazer coisas que estava acostumado a fazer (cozinhar, lavar roupa, trabalhar com ferramentas etc.) por causa da visão?
Sempre
A maioria das vezes
De vez em quando
Poucas vezes

Nunca

23 - Você, por causa da visão, depende do que as outras pessoas falam?
Sempre
De vez em quando..... (3)
A maioria das vezes
Nunca
Poucas vezes 
854 Análise da qualidade de vida de portadores de uveítes de causas infecciosas e não infecciosas pelo questionário NEI-VFQ-25

Continuação da Parte 3

24 - Por causa da sua visão, você tem precisado da ajuda dos outros?
Sempre
A maioria das vezes
De vez em quando
Poucas vezes.

Nunca

25 - Por causa da sua visão, você tem tido receio de fazer algumas coisas com medo de passar vergonha, p.ex. entrar no banheiro errado, não falar com pessoas conhecidas, urinar fora do sanitário, etc.?

Sempre

A maioria das vezes

De vez em quando

Poucas vezes.

Fonte: O questionário NEI-VFQ-25 é um produto da análise e redução de itens do NEI-VFQ-51 (MANGIONE et al. 2001)(15).

Este questionário NEI-VFQ-25 foi traduzido para o português e submetido à adaptação cultural (FERRAZ et al. 2002)(12). 